\title{
X-Ray Absorption Near Edge Spectroscopy of Sulfur in Biomolecules: Two Examples from Glutathione and Insulin
}

\author{
G. Cinque $^{a}$, G. Bellisola ${ }^{b}$, M. Colombatti ${ }^{b}$
}

AND E. BURATTINI ${ }^{c}$

${ }^{a}$ I.N.F.N. - Laboratori Nazionali di Frascati, via Fermi 40, 00044 Frascati, Italy

${ }^{b}$ Department of Pathology, Section of Immunology, University of Verona

Policlinico GB Rossi, P. le L.A. Scuro, 10, 37134 Verona, Italy

${ }^{c}$ Department of Informatics, Faculty of Science, University of Verona

Ca' Vignal 2, 37134 Verona, Italy

\begin{abstract}
Although a minor constituent of cell and tissues, sulfur is an essential element to fulfil a wide range of biological processes, and it is present in the functional groups of many biomolecules that participate to redox reactions in vivo. Cysteine, one of the two S-containing aminoacids present in proteins, contains sulfur in fully reduced form and its thiol group can undergo a range of reactions under physiological conditions. X-ray absorption spectroscopy represents a unique tool to speciate the redox state of sulfur in biomolecules because of the known strong correlation between oxidation state and absorption edge energy shift (over $10 \mathrm{eV}$ ). Moreover, a rich $\mathrm{X}$-ray absorption near edge structure is related to the chemical structures of S-containing biomolecules, as well as significant spectral changes due to biochemical action. The formation of a disulfide bond, i.e. a covalent linkage between the $\mathrm{S}$ atoms of two cysteine residues, or its reduction were investigated only indirectly in biomolecules. X-ray absorption spectroscopy experiments at the sulfur $K$-edge were performed at the soft X-ray beamline in Frascati using the wiggler source of the $0.51 \mathrm{GeV}$ storage ring DAФNE. $\mathrm{X}$-ray absorption near edge structure data were collected to distinguish in situ between S-thiol and disulfide on model protein systems. Such preliminary results confirm this technique as a unique probe of sulfur chemistry in vivo. Quantitative speciation of S-metabolites can be foreseen in biological tissues with no chemical manipulations of the specimen.
\end{abstract}

PACS numbers: 78.70.Dm, 87.64.Gb 


\section{Introduction}

Sulfur is endowed by an electronic configuration (Ne) $3 s^{2} 3 p^{4}$, thus allowing chemical valence states between +6 and -2 . In living cell, this key element is mainly contained in methionine and cysteine aminoacids [1]. In cysteine, this element is fully reduced (valence state -2 assigned), and its thiol group spans a variety of physiological reactions. Thiol/disulfide-exchange reactions are nucleophilic substitutions of a thiol or thiolate nucleophile ( $\mathrm{RSH}$ or $\mathrm{RS}^{-}$) on a disulfide bond (RS-SR), which lead to the formal oxidation of the nucleophile and reduction of the "exchanged" leaving group. These are among the most common redox pathways of cysteine residues in vivo [2].

Glutathione ( $\gamma$-Glu-Cys-Gly or GSH) is a vital intra- and extracellular mammalian tripeptide that participates in many important biochemical processes controlling the homeostasis of the cells. The role of GSH as a key regulator of thiol redox intracellular balance has been clearly established: this revealed that GSH is essential in regulating the cell's life cycle, and that the reduction of intracellular GSH parallels cell death by apoptosis and necrosis. GSH $\left[E^{0^{\prime}}\right.$ (GSSG/2GSH) $\approx-250 \mathrm{mV}]$ is readily oxidized to glutathione disulfide (GSSG) as described by the reaction

$$
4 \mathrm{GSH}+\mathrm{O}_{2} \leftrightarrow 2 \mathrm{GSSG}+2 \mathrm{H}_{2} \mathrm{O} .
$$

By this mean the reducing agent $\mathrm{H}$ is freed and activated within the cell, while the glutathione molecule goes through the two configurations of Fig. 1.
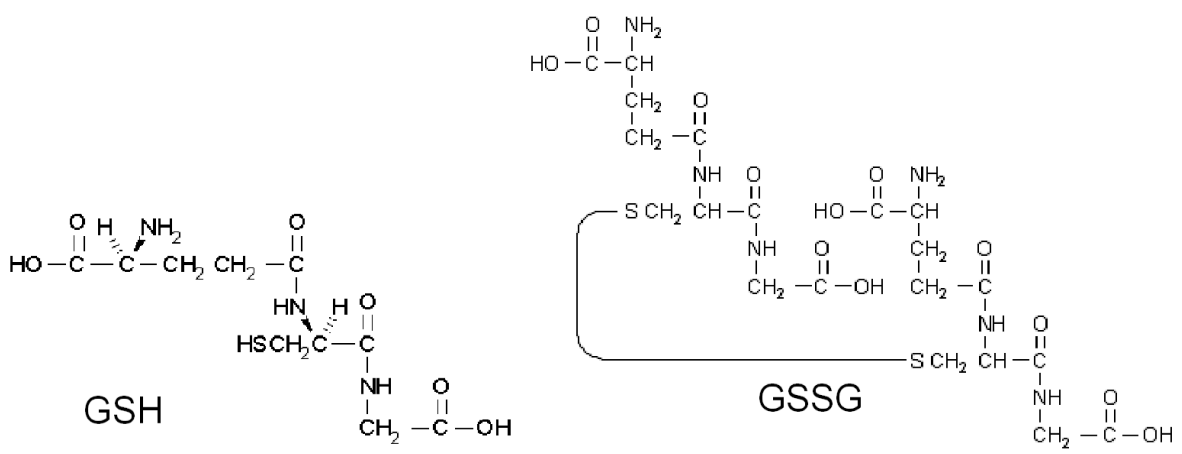

Fig. 1. Tripeptide glutathione in the reduced (GSH) or the oxidized (GSSG) forms.

The GSH/GSSG ratio adjusts the global intracellular thiol redox potential. Most healthy cells have a GSH/GSSG ratio in the range of 100:1 [2]. Such ratio decreases during oxidative stress, and disturbance of the GSH/GSSG ratio may affect cellular proteins, DNA, and membranes. Moreover, this parameter could be different between normal and tumor cells or tissues.

Cysteine in proteins participates in a range of different redox reactions. Very important is the formation of a disulfide bond, i.e. a covalent linkage between the 
$\mathrm{S}$ atoms of two cysteine residues. This bond is often essential to the protein structural stability and folding, as exemplified by 3 disulfides in the insulin molecule.

Different techniques are available for biological speciation analysis of bioorganic sulfur, including sensitive ion conductivity plasma mass spectroscopy (ICP-MS) detection of non-metals coupled to capillary nanoflow high performance liquid chromatography (HPLC) or capillary electrophoresis, laser ablation ICP-MS detection of proteins in gel electrophoresis, and isotope dilution quantification [3].

The conformational change by formation of a covalent linkage between two $\mathrm{S}$ atoms from cysteine residues, or its reduction, has been not directly established in biomolecules, whereas X-ray absorption spectroscopy (XAS) might represent a unique tool to speciate the redox state of $\mathrm{S}$ element in biomolecules because of the known strong correlation between its oxidation state and absorption edge energy shift. Moreover, a rich X-ray absorption near edge structure (XANES) is related to chemical structure, and within the same molecule to its conformational states, of S-containing biosystems. The present work focuses on the possibility to distinguish between sulfydril and disulfide in proteins by XAS.

\section{Materials and methods}

$\mathrm{XAS}$ at the sulfur $K$-edge was performed at the soft X-ray beamline in Frascati using the wiggler source of the $0.51 \mathrm{GeV}$ storage ring DA $\Phi$ NE [4].

Ultrapure glutathione GSH $\left(M_{r}=307.3 \mathrm{Da}\right)$, GSSG $\left(M_{r}=612.6 \mathrm{Da}\right)$ and bovine pancreas insulin $\left(M_{r}=5733.49 \mathrm{Da}\right)$ were dissolved in $100 \mathrm{mM}$ potassium phosphate buffer, $\mathrm{pH}=7.2$. Several $20 \mu \mathrm{L}$ aliquots of each solution were deposited and dried on $8 \mu \mathrm{m}$ thick paper filters in order to obtain an equivalent absolute content between few $\mathrm{mg}$ ( 1 to $3 \mathrm{mg}$ in the case of GSSG and GSH, and at maximum $10 \mathrm{mg}$ for insulin) of elemental S over an irradiated area of $2 \times 10 \mathrm{~mm}^{2}$. Native sulfur powder was finely ground, then stacked on (both pages of) paper for the elemental sulfur reference, while the same support was stained by a nickel sulfate saturated water solution to get an oxidized S standard.

XANES measures were collected in transmission in single runs of maximally 20 min and acquisition time between 1 to 2 s per energy point [5]. Si(111) or Ge(111) crystal couples in the "boomerang" fixed-exit monochromator allowed an energy resolution between 0.2 to $0.4 \mathrm{eV}$ (i.e. $E / \Delta E$ better than 1000 at the $K$-edge of S). Constant energy steps smaller than the energy resolution were always used, thus spectra over-sampling on all data acquired was generally achieved. Standard detection system by ion chambers - filled with either Ar or $\mathrm{N}_{2}$ gas, and electronic chain with amplification $10^{10} \mathrm{~V} / \mathrm{A}$ - was used.

\section{Results}

The experimental results of XAS spectroscopy in transmission mode are reported in Fig. 2. Here the 3 graphs show an enlarged XANES region around the sulfur $K$-edge, with respect to the $100 \mathrm{eV}$ window normally used. The native sulfur 


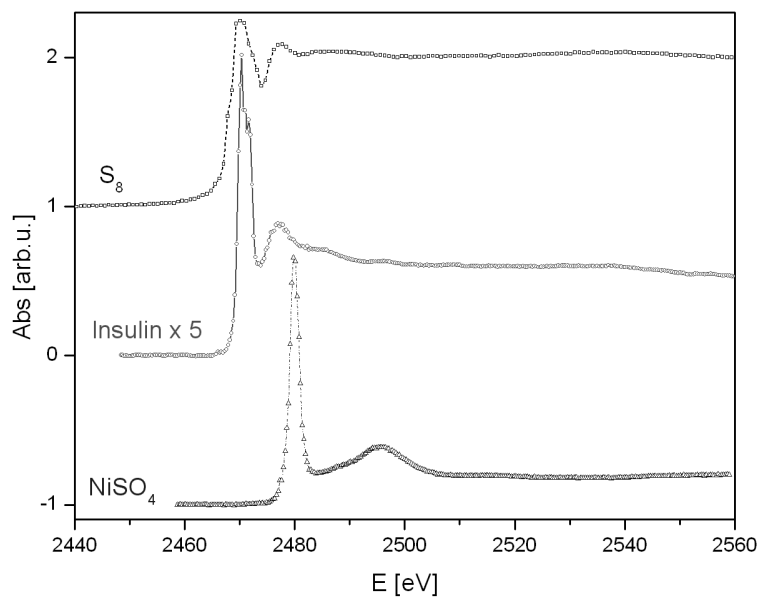

Fig. 2. XANES measured in transmission of native sulfur powder, insulin protein and nickel sulfate solutions all deposited on paper support.

$\left(\mathrm{S}_{8}\right)$ spectrum, with its typical atomic-like profile by one main peak and other resonances merging into a step transition, is used as reference for the energy scale calibration (S K-shell binding energy $2472 \mathrm{eV}$ ) [6]. This spectrum is associated to the chemically reduced form of sulfur. On the opposite side, the $\mathrm{NiSO}_{4}$ spectrum clearly demonstrates the whole spectrum shift $(\approx 10 \mathrm{eV})$ in energy due to the valence state changing, namely toward the most oxidized form of $\mathrm{S}$ atom associated to the sulfate anion. In between, the XANES spectrum of the 6 sulfur bond atoms present in the insulin polypeptide has been multiplied 5 times for the sake of clarity. The position of the energy threshold clearly indicates the reduced state of the $\mathrm{S}$ atoms within the insulin protein, as expected.

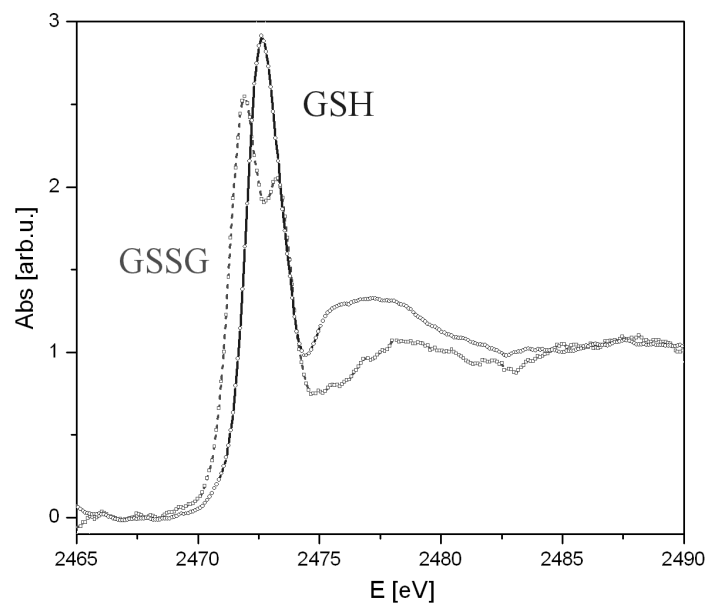

Fig. 3. XANES measured in transmission of reduced (GSH) or oxidized (GSSG) glutathione on paper support. 
XANES data have been collected on model reducing substrate and protein systems, namely glutathione and insulin, also to distinguish between thiol sulfur and disulfide. In particular, Fig. 3 refers to the XANES measurements on glutathione tripeptide in both its oxidized (GSSG with one S-S bond) and reduced (GSH with $1 \mathrm{~S}$ atom) configurations. In the strictly defined XANES energy range, a significant change in the $\mathrm{S}$ absorption $K$-edge accompanies the formation (or not) of the disulfur bond. Following [7], the analysis of near-edge spectra of oxidized glutatione reveals two clearly resolved peaks, separated by more than $c a$. $1.5 \mathrm{eV}$, which can be assigned to $1 s \rightarrow \sigma^{*}(\mathrm{~S}-\mathrm{S})$ and $1 s \rightarrow \sigma^{*}(\mathrm{~S}-\mathrm{C})$, respectively, for the lower and higher energy electronic transition. This is also experimentally confirmed in the case of insulin in Fig. 2, where the double peak structure of the XANES profile is assigned to the three disulfides and equal number of double cystein bonds.

\section{Conclusions}

XAS is a powerful tool nowadays widely applied in biophysics to investigate metallic sites of proteins and enzymes. The $\mathrm{S}$ elemental content in biological system, and in vivo tissues/fluids, is generally in the non-negligible range of minor elements (few \% or below): in this sense the XANES technique is privileged due to the major signal variation (10 to 50\%) of the X-ray absorption edge. The speciation of sulfur in biological molecules is privileged because of the intrinsic XANES sensitivity to local electronic and chemical environment, as well as to coordination state of the absorbing atom. In fact, different spectra are mostly used as fingerprints of the chemical forms in which the sulfur is present. Disulfide bond formation plays a key role in protein folding and as mechanism to go through structural and conformational changes: sulfur XANES uniqueness resides in its capacity to distinguish straightforwardly the reduced (thiolate) and oxidized (disulfide) forms of proteins. Such preliminary results on glutathione and insuline, biological benchmark systems, confirm XANES technique as a direct probe of disulfur bond as well as S chemistry at protein concentration of few millimoles, i.e. close to the in vivo content, notwithstanding in transmission mode. Its use for quantitative speciation of S-metabolites is attractive since no chemical manipulations of the biological specimens are required. Secondary X-ray fluorescence detection will certainly improve the detection limit, thus allowing a lower protein amount to be measured and accessing larger protein complexes with few disulfides but higher biomedical interest. The future challenge is the possibility of performing site-specific sulfur XANES, but this requires detecting different emission lines by higher resolution fluorescence spectroscopy.

\section{Acknowledgments}

Special thanks to Antonio Grilli and Agostino Raco for their help in the experimental setup at the DA $\Phi$ NE-L Lab of Frascati. 
This project is partially supported by Fondazione CariVerona, grant 2003 "Integrazione tra tecnologia e sviluppo di settore", project "Caratterizzazione strutturale e funzionale di cellule e tessuti sani e tumorali mediante spettroscopia e spettromicroscopia infrarossa con sorgenti convenzionali e con Luce di Sincrotone per lo sviluppo di nuove tecniche di diagnosi precoce dei tumori nell'uomo".

\section{References}

[1] H. Sies, R. Brigelius, T.P.M. Ackerboom, in: Functions of Glutathione: Biochemical, Physiological, Toxicological, and Clinical Aspects, Eds. A. Larsson, S. Orrenius, A. Holmgren, B. Mannervik, Raven Press, New York 1983, p. 231.

[2] H.F. Gilbert, in: Advances in Enzymology, Vol. 63, Ed. A. Meister, Wiley, New York 1990, p. 69.

[3] J. Szpunar, Analyst 130, 442 (2005).

[4] E. Burattini, G. Cinque, S. Dabagov, A. Grilli, A. Marcelli, F. Monti, E. Pace, M. Piccinini, A. Raco, in: Proc. 8th Int. Conf. SRI 2003, Eds. T. Warwick, J. Arthur, H.A. Padmore, J. Stöhr, AIP Conf. Proc., San Francisco 2004, Vol. 705 , p. 81.

[5] G. Cinque, E. Burattini, A. Grilli, S. Dadagov, in: Int. Conf. on Charged and Neutral Particles Channeling Phenomena, Channeling 2004, Ed. S.B. Dabagov, SPIE Proc. 2005, Vol. 5974, INFN, Lab. Nazionali di Frascati (Italy) and RAS, P.N. Lebedev Physical Institute (Russia), 2005, p. 448.

[6] G.P. Williams, in: X-Ray Data Booklet, Eds. A.C. Thompson, D. Vaughan, Lawrence Berkeley National Laboratory, Berkeley 2001, Sec. 1.1.

[7] I.J. Pickering, R.C. Prince, T. Divers, G.N. George, FEBS Lett. 441, 11 (1998). 